\title{
Carcinomatous Pericarditis in 3 Breast Cancer Patients with Long-Term Survival
}

\author{
Takanori Konishi $^{\text {a }}$ Rikiya Nakamura ${ }^{a}$ \\ Naohito Yamamoto ${ }^{a}$ Yasuhide Onai $^{\mathrm{a}}$ Toshi Okada $^{\mathrm{a}}$ \\ Makiko Itami ${ }^{b}$ Masaru Miyazaki ${ }^{\mathrm{c}}$ \\ Divisions of ${ }^{a}$ Breast Surgery and ${ }^{b}$ Diagnostic Pathology, Chiba Cancer Center \\ Hospital, and ${ }^{\mathrm{C} D e p a r t m e n t}$ of General Surgery, Chiba University Graduate \\ School of Medicine, Chiba, Japan
}

\section{Key Words}

Breast cancer - Carcinomatous pericarditis - Cardiac tamponade - Pericardiocentesis

\begin{abstract}
With advances in drug treatment of breast cancer, the number of patients experiencing cardiac toxicity or carcinomatous pericarditis is expected to increase. These conditions can cause cardiac tamponade, which is a potentially fatal condition requiring prompt diagnosis and treatment. We experienced 3 breast cancer patients with cardiac tamponade due to carcinomatous pericarditis who survived for prolonged periods after treatment with pericardiocentesis and intrapericardial instillation. The 3 women were 68,46 and 46 years old, respectively, and receiving treatment for recurrent breast cancer after surgery. They developed dyspnea and cough and were diagnosed with cardiac tamponade by echocardiography. Pericardiocentesis was performed, and cytology of the effusion confirmed the diagnosis of carcinomatous pericarditis. Intrapericardial instillation of cisplatin reduced the cardiac effusion, ameliorating symptoms. The patients died 13,31 and 14 months later, respectively. In our clinical review of 13 other cases of cardiac tamponade due to breast cancer, $85 \%$ achieved local control after the aforementioned local treatments, which were considered to be effective. Although the overall prognosis was poor with a median survival time of only 4 months, some patients were able to survive more than 1 year after local treatment with subsequent systemic therapy.
\end{abstract}




\section{Introduction}

Recent developments in molecular targeting agents and new anticancer drugs have contributed to dramatic improvements in the life expectancy of patients with recurrent breast cancer [1, 2]. With the prolongation of survival, long-term treatment for recurrent cancer has become more frequent and the importance of systemic management has increasingly been recognized. It is particularly expected that the occurrence of cardiac tamponade due to cardiac toxicity associated with the use of molecular targeting agents or carcinomatous pericarditis will increase. Therefore, it is important to assure early diagnosis and appropriate treatment of heart failure in these patients [3]. We experienced 3 breast cancer patients with cardiac tamponade due to carcinomatous pericarditis who survived for a prolonged period after treatment with pericardiocentesis and intrapericardial instillation of cisplatin (CDDP). We retrospectively reviewed therapeutic outcomes of our patients, including 13 additional patients, who developed cardiac tamponade, to determine the usefulness of pericardiocentesis for carcinomatous pericarditis.

\section{Case Reports}

Case 1

A 60-year-old woman underwent breast-conserving surgery and sentinel lymph node biopsy for left breast cancer. Histological examination showed a scirrhous carcinoma $1.6 \mathrm{~cm}$ in diameter (n0, ER positive, PgR positive and HER2 negative). Postoperative radiotherapy and tamoxifen were administered as adjuvant therapy. Four years and 8 months after surgery, she developed carcinomatous pleurisy, for which anastrozole treatment was started. After that, she had hepatic metastasis and peritoneal dissemination, for which capecitabine, paclitaxel and irinotecan were administered. Seven years and 9 months after surgery, when she was 68 years old, the patient developed dyspnea, and the presence of pericardial effusion was confirmed by echocardiography. Diuretics were administered but the symptoms worsened, and the patient was admitted to the hospital.

At the time of admission, her blood pressure was $107 / 70 \mathrm{~mm} \mathrm{Hg}$, pulse rate $84 / \mathrm{min}$ and $\mathrm{SpO}_{2} 97 \%$ (room air). Chest X-ray showed an enlarged heart shadow (fig. 1a) and chest CT showed pericardial effusion $(1.6 \mathrm{~cm}$ ) and right pleural effusion (fig. 1b). Echocardiography revealed pericardial effusion and collapse of the right heart (fig. 1c), so cardiac tamponade was diagnosed. Pericardiocentesis was performed and a drainage tube was placed. Bloody pericardial fluid was drained, and the cytology was interpreted as class V. CDDP $10 \mathrm{mg}$ was administered into the pericardial cavity on the 3rd hospital day, and the drainage tube was removed on the 4th hospital day. After discharge, the patient showed improvements in her activities of daily living (ADL) and restarted irinotecan. She then developed carcinomatous peritonitis, for which CDDP was administered into the abdominal cavity. Eight years and 10 months after surgery, the patient died (1 year and 1 month after the onset of cardiac tamponade).

Case 2

A 33-year-old woman underwent mastectomy of the right breast and axillary lymph node dissection for right breast cancer. Then, she underwent mastectomy of the left breast and axillary lymph node dissection for left breast cancer at age 38 years. Histological examination showed a solidtubular carcinoma $4.0 \mathrm{~cm}$ in diameter (n1, ER negative, PgR positive and HER2 negative). Postoperative adjuvant treatment with tamoxifen and doxifluridine was administered. Lung metastasis was found 5 years and 5 months after surgery, for which CMF (cyclophosphamide + methotrexate + fluorouracil) and anastrozole were administered. Seven years and 5 months after surgery, when she was 46 years old, she developed cough and dyspnea. Chest CT showed pericardial effusion $(2 \mathrm{~cm})$ and multiple lung metastases. The patient was diagnosed with cardiac tamponade by echocardiography and admitted to the hospital. 
Pericardiocentesis was performed and a drainage tube was placed. Bloody pericardial fluid was drained, and the cytology was interpreted as class V. CDDP $10 \mathrm{mg}$ was administered into the pericardial cavity on the 2 nd hospital day, and the drainage tube was removed on the 4 th hospital day. After discharge, the patient showed improvements in her ADL and started exemestane. She then developed lung metastases, for which capecitabine was administered. She also developed brain metastasis, for which tumorectomy and whole-brain radiation were performed. Ten years after surgery for left breast cancer, the patient died (2 years and 7 months after the onset of cardiac tamponade).

Case 3

A 39-year-old woman underwent mastectomy and axillary lymph node dissection for left breast cancer. Histological examination showed a scirrhous carcinoma $2.5 \mathrm{~cm}$ in diameter (n1, ER positive, PgR positive and HER2 negative). Postoperative adjuvant treatment with tamoxifen and an LH-RH agonist was administered. Six years and 6 months after surgery, she developed a cervical lymph node recurrence, for which an LH-RH agonist and docetaxel were administered. Nine years and 7 months after surgery, when she was 46 years old, she developed dyspnea, and pericardial effusion $(1.1 \mathrm{~cm})$ and right pleural effusion were confirmed by echocardiography. The patient was admitted to the hospital with a diagnosis of cardiac tamponade.

Pericardiocentesis was performed and a drainage tube was placed. The cytology was interpreted as class IIIb. Carcinomatous pericarditis was strongly suspected from the clinical course. CDDP $10 \mathrm{mg}$ was administered into the pericardial cavity on the 5 th hospital day, and the drainage tube was removed on the 7th hospital day. After discharge, the patient showed improvements in her ADL and started EC (epirubicin + cyclophosphamide). She had an exacerbation of the cervical lymph node metastasis, for which capecitabine was administered. She also developed brain metastasis, for which whole-brain radiation therapy was performed. Ten years and 9 months after surgery, the patient died (1 year and 2 months after the onset of cardiac tamponade).

\section{Discussion}

Carcinomatous pericarditis is observed in $10-20 \%$ of patients with malignancies, and the common cancers are lung cancer, breast cancer, leukemia and malignant lymphoma. Carcinomatous pericarditis is detected at autopsy in $10-20 \%$ of breast cancer patients, although the prevalence of this condition during the clinical course is relatively low at $6.1 \%[4,5]$. Cardiac tamponade is a life-threatening condition requiring prompt diagnosis and treatment.

Subjective symptoms characterized by dyspnea and cough are useful for making an early diagnosis of cardiac tamponade. In addition, the characteristic physical findings include paradoxical pulse, carotid distention, edema and decreased heart sounds. Heart enlargement and pleural effusion are seen on chest X-ray and decreased voltage and tachycardia on electrocardiography. In particular, echocardiography is a simple and useful diagnostic tool that allows a definitive diagnosis of pericardial effusion, right heart collapse and decreased wall motion. Pericardial effusion is caused by a variety of conditions including carcinomatous pericarditis, drug therapy with molecular targeting agents or anticancer drugs, radiation therapy, inflammation and so on. Although cardiac dysfunction caused by molecular targeting agents is usually reversible and often resolves after cessation of administration [6], carcinomatous pericarditis requires aggressive treatment. Pericardial fluid cytology is useful in the differential diagnosis of carcinomatous pericarditis with a reported sensitivity of $90 \%$ [7]. In addition, coexisting carcinomatous pleurisy or lung metastasis is observed in $63-100 \%$ of patients, so physicians should be alert to the possible presence of such conditions [8]. 
Treatments of cardiac tamponade include 'pericardiocentesis and intrapericardial instillation' and 'pericardial window', and no difference in recurrence rates between the two methods has been reported [9]. Pericardiocentesis and intrapericardial instillation is the first choice because of safety and wide availability. Pericardial window should be considered when the condition is intractable. According to Arao et al. [10], indications for this treatment are heart failure symptoms such as severe orthopnea and dyspnea (NYHA class III or IV) and echo-free space of at least $1 \mathrm{~cm}$ observed during the diastolic phase, which ensures the safety of the centesis procedure. Intrapericardial instillation is regarded as an important part of the treatment because the recurrence rate of pericardial effusion approached $70 \%$ when only pericardiocentesis and drainage were performed [11]. The complications of intrapericardial instillation include chest pain, pyrexia, inflammation and constrictive pericarditis. Bleomycin, mitomycin C, CDDP and epirubicin are reportedly effective treatments. The European Society of Cardiology guidelines has recommended the use of thiotepa for the treatment of carcinomatous pericarditis in breast cancer patients [12]. Bischiniotis et al. [13] recommended the use of CDDP for the following: (1) no cardiac toxicity; (2) no pain or hematological toxicity, owing to lower absorption from the pericardium, and (3) no occurrence of constrictive pericarditis. Thus, we chose CDDP in the present 3 patients.

Table 1 shows 13 additional cases of cardiac tamponade encountered in our hospital. All 13 patients had recurrent carcinomas. HER2 overexpression was observed in 1 of $10(10 \%)$ patients. Trastuzumab was not used in any of these patients, and none of the patients presented with congestive heart failure symptoms associated with the use of molecular targeting agents. The average time period between the first recurrence and the diagnosis of pericardial effusion was 34.7 months (range 3-90). Many patients had been treated with anticancer drugs for a prolonged period, so differential diagnosis from drug-induced heart failure was required. Pericardial fluid cytology was performed in 12 patients, and 10 patients were diagnosed with carcinomatous pericarditis. The remaining patients were also strongly suspected of having carcinomatous pericarditis based on their clinical courses and the presence of bloody pericardial fluid.

The procedure of pericardiocentesis and intrapericardial instillation employed in our hospital is shown in fig. 2. We perform pericardiocentesis under ultrasonic guidance using a subxiphoid approach or an intercostal approach. The complications of pericardiocentesis include ventricular perforation, ventricular arrhythmia and pneumothorax. Thus, this procedure must be performed by a sufficiently trained doctor. Intrapericardial instillation should be administered after the pericardial effusion has been drained in patients with carcinomatous pericarditis confirmed by cytology. CDDP was used for 11 of the reviewed patients, and CDDP + OK-432 for the other 2 patients. In most of these cases, pericardial effusion was markedly reduced after drug administration and the drainage tubes were subsequently removed. When the pericardial effusion was not reduced by treatment, drug administration was repeated. No recurrence of pericardial effusion was observed in 11 patients (85\%) after the initial local treatment. In the remaining 2 patients who showed recurrence of pericardial effusion, repeat treatment with the same drug was effective. Therefore, pericardiocentesis and intrapericardial instillation of CDDP were considered to be effective for local control. 
Cardiac tamponade as the initial manifestation of recurrence of breast cancer is rare [8]. This condition is occasionally observed in patients with aggravation of recurrent breast cancer. For the 13 patients reviewed herein, the median survival time after the onset of cardiac tamponade was only 4 months (fig. 3 ). Eight patients (62\%) died within 6 months, and 6 of the 8 patients showed no improvements in ADL after local control. These 6 patients were considered to be in the end stage of breast cancer at the time they developed carcinomatous pericarditis. On the other hand, there are a few case reports of long-term survival after local treatment [14]. All 3 patients in the present study survived more than 1 year after the onset of cardiac tamponade and also showed improvements in ADL after receiving local treatment. They were able to receive systemic drug treatment: irinotecan in case 1 , exemestane and capecitabine in case 2 , and capecitabine in case 3 . It is difficult to predict outcomes at the time of cardiac tamponade onset because general conditions are poor due to acute heart failure symptoms. However, for the cases whose distant metastases are controlled by drug treatment, local treatment not only leads to the relief of symptoms but also makes it possible to conduct subsequent systemic therapy and prolong survival time.

\section{Conclusion}

Pericardiocentesis and intrapericardial instillation of CDDP are considered to be effective local treatments for cardiac tamponade due to carcinomatous pericarditis. We have described 3 breast cancer patients with cardiac tamponade who showed improvements in ADL after local treatment and achieved long-term survival with subsequent systemic treatment.

Table 1. Cases of malignant effusion in breast cancer

\begin{tabular}{|c|c|c|c|c|c|c|c|c|c|c|c|}
\hline \multirow[t]{2}{*}{ Case } & \multirow[t]{2}{*}{ Age } & \multirow[t]{2}{*}{ ER } & \multirow[t]{2}{*}{ PgR } & \multirow[t]{2}{*}{ HER2 } & \multicolumn{2}{|c|}{ Pericardial effusion } & \multirow{2}{*}{$\begin{array}{l}\text { Intrapericardial } \\
\text { sclerosis }\end{array}$} & \multirow{2}{*}{$\begin{array}{l}\text { Improve- } \\
\text { ment of } \\
\text { ADL }\end{array}$} & \multirow{2}{*}{$\begin{array}{l}\text { Recurrence } \\
\text { of } \\
\text { pericardial } \\
\text { effusion }\end{array}$} & \multirow{2}{*}{$\begin{array}{l}\text { Systemic } \\
\text { therapy } \\
\text { after } \\
\text { tamponade }\end{array}$} & \multirow{2}{*}{$\begin{array}{l}\text { Survival } \\
\text { time after } \\
\text { tamponade } \\
\text { months }\end{array}$} \\
\hline & & & & & characte & cytology & & & & & \\
\hline 1 & 68 & + & + & - & bloody & class $\mathrm{V}$ & CDDP $10 \mathrm{mg}$ & + & - & + & 13 \\
\hline 2 & 46 & + & + & - & bloody & class $\mathrm{V}$ & CDDP $10 \mathrm{mg}$ & + & - & + & 31 \\
\hline 3 & 46 & + & + & - & - & class IIIb & CDDP $10 \mathrm{mg}$ & + & - & + & 14 \\
\hline 4 & 33 & - & + & unknown & bloody & class $\mathrm{V}$ & CDDP $10 \mathrm{mg}$ & - & + & - & 3 \\
\hline 5 & 61 & + & + & unknown & bloody & class $\mathrm{V}$ & $\begin{array}{l}\text { CDDP } 10 \mathrm{mg}+ \\
\text { OK-432 5KE }\end{array}$ & - & - & - & 1 \\
\hline 6 & 54 & - & - & + & bloody & class $\mathrm{V}$ & $\begin{array}{l}\text { CDDP } 10 \mathrm{mg}+ \\
\text { OK-432 5KE }\end{array}$ & - & - & - & 1 \\
\hline 7 & 54 & + & + & unknown & bloody & unknown & CDDP 10 mg & + & - & + & 4 \\
\hline 8 & 51 & - & - & - & bloody & class $\mathrm{V}$ & CDDP $10 \mathrm{mg}$ & - & - & - & 1 \\
\hline 9 & 46 & + & + & - & bloody & class $\mathrm{V}$ & CDDP $20 \mathrm{mg}$ & + & + & + & 8 \\
\hline 10 & 51 & - & - & - & serous & class IIa & CDDP $20 \mathrm{mg}$ & - & - & - & 5 \\
\hline 11 & 56 & + & + & - & bloody & class V & CDDP $10 \mathrm{mg}$ & - & - & - & 7 \\
\hline 12 & 60 & + & + & - & bloody & class $\mathrm{V}$ & CDDP $10 \mathrm{mg}$ & + & - & - & 4 \\
\hline 13 & 74 & + & - & - & bloody & class $\mathrm{V}$ & CDDP $10 \mathrm{mg}$ & - & - & - & 1 \\
\hline
\end{tabular}



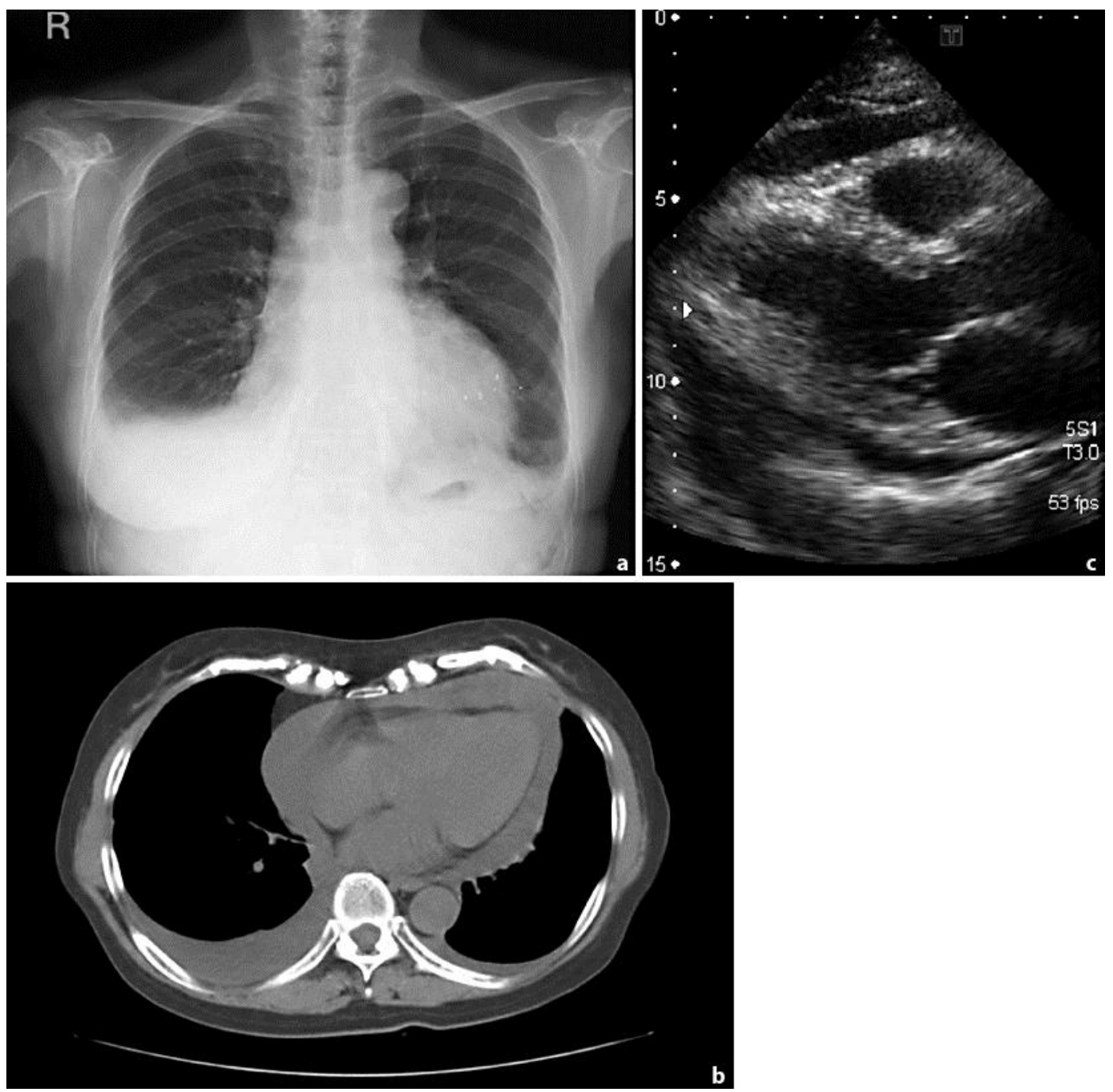

Fig. 1. a Chest X-ray showing enlarged cardiac silhouette. b CT scan showing pericardial effusion and pleural effusion. $\mathrm{c}$ Echocardiogram showing pericardial effusion and right ventricular collapse.

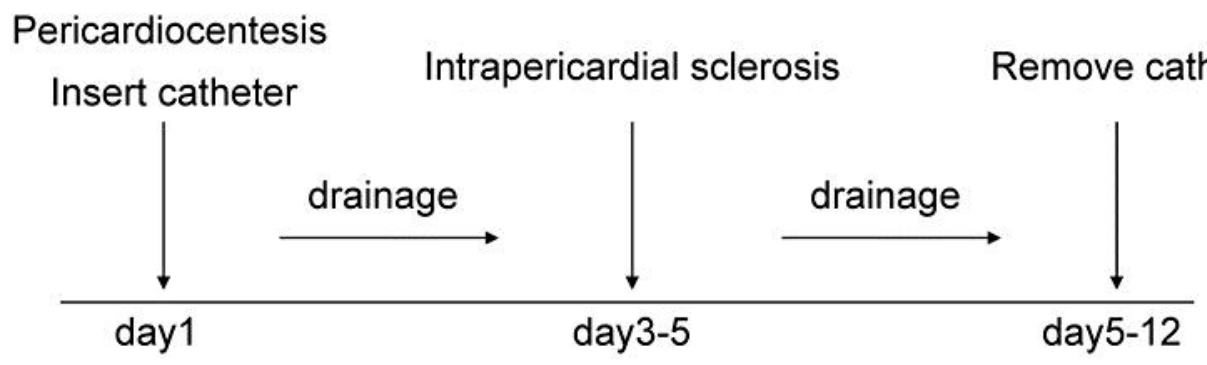

Fig. 2. Method of treatment for malignant pericardial effusion. 


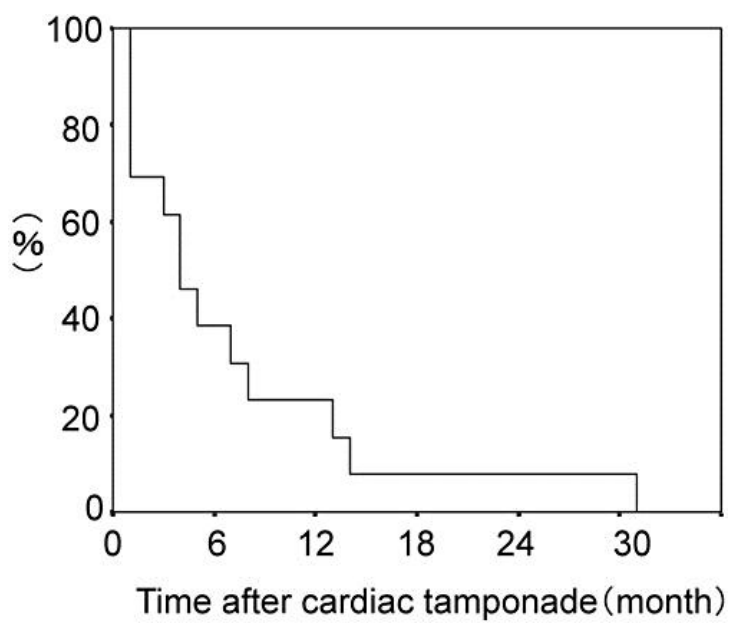

Fig. 3. Overall survival rate of malignant pericardial effusion in breast cancer.

\section{References}

1 Marty M, Cognetti F, Maraninchi D, Snyder R, Mauriac L, Tubiana-Hulin M, et al: Randomized phase Il trial of the efficacy and safety of trastuzumab combined with docetaxel in patients with human epidermal growth factor receptor 2-positive metastatic breast cancer administered as first-line treatment: The M77001 Study Group. J Clin Oncol 2005;23:4265-4274.

-2 Twelves C, Cortes J, Vahdat LT, Wanders J, Akerele C, Kaufman PA: Phase III trials of eribulin mesylate (E7389) in extensively pretreated patients with locally recurrent or metastatic breast cancer. Clin Breast Cancer 2010;10:160-163

3 Slamon DJ, Leyland-Jones B, Shak S, Fuchs H, Paton V, Bajamonde A, et al: Use of chemotherapy plus a monoclonal antibody against HER2 for metastatic breast cancer that overexpresses HER2. N Engl J Med 2001;344:783-792.

4 Buck M, Ingle JN, Giuliani ER, Gordon JR, Therneau TM: Pericardial effusion in women with breast cancer. Cancer 1987;60:263-269.

5 Kimijima I, Abe R: The palliative therapy for breast cancer patients -the characteristics of lung pleural and pericardial metastasis and their management. Jpn J Breast Cancer 1997;12:440-447.

-6 Guarneri V, Lenihan DJ, Valero V, Durand JB, Broglio K, Hess KR, et al: Long-term cardiac tolerability of trastuzumab in metastatic breast cancer: The M.D. Anderson Cancer Center Experience. J Clin Oncol 2006;24:4107-4115.

7 Wilkes JD, Fidias P, Vaickus L, Perez RP: Malignancy-related pericardial effusion. 127 cases from Roswell Park Cancer Institute. Cancer 1995;76:1377-1387.

-8 Swanepoel E, Apffelstaedt JP: Malignant pericardial effusion in breast cancer: terminal event or treatable complication? J Surg Oncol 1997;64:308-311.

9 Girardi LN, Ginsberg RJ, Burt ME: Pericardiocentesis and intrapericardial sclerosis: effective therapy for malignant pericardial effusions. Ann Thorac Surg 1997;64:1422-1427.

10 Arao M, Kitahara Y, Setsuta K: The study of survival time after pericardiocentesis for the patients with carcinomatous pericarditis - the suggestion of a safer method of pericardiocentesis. Heart 2008;40:691697.

11 Hosomi Y, Ookuma Y, Shibuya M: Treatment of malignant pericardial effusion (in Japanese). Jpn J Cancer Chemother 2008;35:906-909.

12 Maisch B, Seferović PM, Ristić AD, Erbel R, Rienmüller R, Adler Y, et al: Guidelines on the diagnosis and management of pericardial diseases executive summary. Eur Heart J 2004;25:587-610.

13 Bischiniotis TS, Lafaras CT, Platogiannis DN, Moldovan L, Barbetakis NG, Katseas GP: Intrapericardial cisplatin administration after pericardiocentesis in patients with lung adenocarcinoma and malignant cardiac tamponade. Hellenic J Cardiol 2005;46:324-329.

14 Ue N, Kawabata H, Ueno T, Hirata M, Tanaka K: Cardiac tamponade due to malignant pericardial effusion in 3 patients with breast cancer. J Jpn Surg Assoc 2002;63:1658-1661. 